\title{
Development and characterization of sugar palm starch and poly(lactic acid) bilayer films
}

\begin{abstract}
The development and characterization of environmentally friendly bilayer films from sugar palm starch (SPS) and poly(lactic acid) (PLA) were conducted in this study. The SPS-PLA bilayer films and their individual components were characterized for their physical, mechanical, thermal and water barrier properties. Addition of 50\% PLA layer onto 50\% SPS layer (SPS50-PLA50) increased the tensile strength of neat SPS film from 7.74 to $13.65 \mathrm{MPa}$ but reduced their elongation at break from 46.66 to $15.53 \%$. The incorporation of PLA layer significantly reduced the water vapor permeability as well as the water uptake and solubility of bilayer films which was attributed to the hydrophobic characteristic of the PLA layer. Furthermore, scanning electron microscopy (SEM) image of SPS50-PLA50 revealed lack of strong interfacial adhesion between the SPS and PLA. Overall, the incorporation of PLA layer onto SPS films enhances the suitability of SPS based films for food packaging.
\end{abstract}

Keyword: $\quad$ Bilayer film; Food packaging; Poly(lactic acid); Sugar palm starch 